\title{
Respuesta superovulatoria en vacas donantes Brahman usando ablación folicular previo a protocolos de superovulación
}

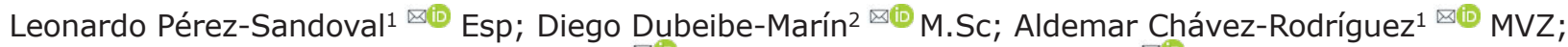 \\ Juan García-Jiménez ${ }^{1} \bowtie \mathbb{B}$ MVZ; Diego Velasco-Acosta ${ }^{1 *} \bowtie \mathbb{C}$ Ph.D.
}

${ }^{1}$ Corporación Colombiana de Investigación Agropecuaria (AGROSAVIA), Km 14 vía Bogotá - Mosquera, Colombia.

2Universidad Federal de Pará, Laboratorio de fertilización In Vitro, Instituto de Ciencias Biológicas, Belém, Pará, Brasil.

*Correspondencia: dvelasco@agrosavia.co

Recibido: Julio 2018; Aceptado: Noviembre 2018; Publicado: Abril 2019.

\section{RESUMEN}

Objetivo. Determinar el efecto de la ablación folicular en el inicio de un protocolo de superovulación (SPO) sobre la respuesta superovulatoria en vacas donantes de raza Brahman. Materiales y métodos. Se utilizaron 20 vacas de raza Brahman, las cuales fueron distribuidas aleatoriamente en dos grupos: Grupo control $(\mathrm{G} 1 ; \mathrm{n}=10)$, la sincronización de la onda de crecimiento folicular fue realizada mediante la combinación de estrógenos (2.5 mg, Benzoato de Estradiol) y progestágenos (1 gr, implante intravaginal); cuatro días después se inició el protocolo de SPO con la hormona folículoestimulante porcina (FSHp); y grupo ablación $(\mathrm{G} 2 ; \mathrm{n}=10)$, se realizó la ablación folicular y un día después se inició el tratamiento de SPO con FSHp. En los dos grupos la colecta de los embriones se realizó siete días después de la primera inseminación artificial. Resultados. El G2 presentó una mayor proporción de embriones de calidad 1 $(p<0.01)$ en comparación con el G1 $(68.60 \%, 31.22 \%)$, mientras que los animales del grupo G1 presentaron una mayor proporción de embriones de calidad $2(43.04 \%, 18.60 \%, \mathrm{p}<0.01)$. Para las variables total de estructuras colectadas, y total de embriones transferibles, no se observaron diferencias significativas $(p>0.05)$. Conclusiones. La ablación folicular aumentó el porcentaje de embriones de calidad 1, sugiriendo que la implementación de esta técnica, como estrategia para sincronizar el inicio de una nueva onda de crecimiento folicular en tratamientos de SPO, mejora la calidad de los embriones producidos en vacas donadoras Brahman.

Palabras clave: Aspiración folicular, bovinos, embrión, reproducción (Fuente: MeSH).

\section{ABSTRACT}

Objective. The objective of this study was to determine the effect of follicular ablation at the beginning of a superovulation protocol (SOP) on the superovulatory response of Brahman donor cows. Materials and methods. Twenty Brahman cows were used, randomly distributed in two groups: control group $(\mathrm{G} 1, \mathrm{n}=10)$, synchronization of the follicular growth wave was performed by the combination of estrogens ( $2.5 \mathrm{mg}$, estradiol benzoate) and progestagens ( $1 \mathrm{gr}$ intravaginal implant); four days after starting the SOP with porcine follicle stimulating hormone (FSHp); and the ablation group $(\mathrm{G} 2, \mathrm{n}=10)$, follicular ablation was performed and one day after, the SOP treatment with FSHp was initiated. In both groups, embryo collection was performed seven days after the first artificial insemination. Results. The $G 2$ had a higher proportion of quality 1 embryos ( $p<0.01)$ compared to $\mathrm{G} 1(68.60 \% \mathrm{vs}$. $31.22 \%)$, while animals of G1 group had a higher proportion of quality 2 embryos $(43.04 \%$ vs. $18.60 \%, p<0.01)$. For the total of structures collected and the total of transferable embryos, no significant differences were observed $(p>0.05)$. Conclusions. Follicular ablation increased the percentage of quality 1 embryos, suggesting that the implementation of this technique, as a strategy to synchronize the beginning of a new wave of follicular growth when using SOP, improve embryo quality in Brahman donor cows.

Keywords: Bovine, embryo, follicular aspiration, reproduction (Fuente: MeSH).

Como citar (Vancouver)

Pérez-Sandoval L, Dubeibe-Marin D, Chavez-Rodriguez A, Garcia-Jimenez J, Velasco-Acosta D. Respuesta superovulatoria en vacas donantes Brahman usando ablación folicular previo a protocolos de superovulación. Rev MVZ Cordoba. 2019; 24(2):7203-7208. DOI: https://doi.org/10.21897/rmvz.1309

(C)El (los) autor (es), Revista MVZ Córdoba 2019. Este artículo se distribuye bajo los términos de la licencia internacional Creative Commons Attribution 4.0 (https://creativecommons.org/licenses/by-sa/4.0/), que permite el uso sin restricciones, la distribución y la reproducción en cualquier medio, siempre que se otorgue el crédito apropiado al autor o autores originales y la fuente. 


\section{INTRODUCCIÓN}

La técnica de superovulación (SPO) para la producción y transferencia de embriones (TE), ha sido una de las biotecnologías de mayor difusión en programas de mejoramiento genético aplicados a la especie bovina (1). No obstante, las tasas de producción de embriones han permanecido relativamente estáticas a través del tiempo (6.2 embriones viables colectados por donante) (2). La variabilidad en la respuesta a los tratamientos de SPO por las donadoras de embriones, continúa siendo el mayor problema en los programas comerciales de TE (3). Esta variabilidad podría estar relacionada a factores extrínsecos, ligados al proprio tratamiento de $\mathrm{SPO}$, o principalmente, a factores intrínsecos, propios de la dinámica folicular de cada donante, tales como el tamaño de la población folicular, o el número de ondas foliculares, entre otros (4).

Las variaciones en la dinámica folicular son un factor importante en los tratamientos de SPO. Tratamientos de SPO que no inician en el momento de la emergencia de una onda folicular tienen una reducción significativa de la respuesta superovulatoria (5). Adicionalmente, el número de cuerpos lúteos, estructuras colectadas y embriones transferibles disminuye en vacas sometidas a tratamientos de SPO que presentan un folículo dominante (FD) al momento de iniciar el tratamiento, hecho que se relaciona a la capacidad del FD de inducir el proceso de atresia en los folículos subordinados, afectando de esa forma su calidad y capacidad de desarrollo (6). De esa forma, la implementación de estrategias para manipular la dinámica folicular y sincronizar el inicio de la emergencia de la onda folicular con el inicio de los tratamientos de SPO, son determinantes en la intención de reducir la variabilidad de los protocolos de SPO (7).

La manipulación de la dinámica folicular para evitar la presencia de folículos dominantes, tradicionalmente es realizada a través de tratamientos hormonales con asociación de estrógenos y progestágenos (8). Otros tratamientos consisten en la aplicación de hormona liberadora de gonadotrofinas (GnRH), hormona luteinizante (LH), gonadotrofina coriónica humana (hCG) o sus análogos, en cualquiera de estos casos, la emergencia de la nueva onda folicular ocurrirá entre 24 y 48 horas después del tratamiento, dependiendo de la hormona utilizada, y la sincronización solo será efectiva en los animales que ovulen como respuesta al tratamiento $(6,9)$. También se ha demostrado que la sincronización de la emergencia de la onda folicular puede ser inducida de forma mecánica a través de la ablación folicular, aspirando todos los folículos presentes, únicamente folículos $\geq 5 \mathrm{~mm}$ de diámetro, o removiendo el folículo dominante, en cualquiera de los casos, la emergencia de la nueva onda folicular ocurre aproximadamente entre 24 y 48 horas después $(10,11)$.

Aunque han sido relatadas algunas diferencias en las características de la fisiología reproductiva entre ejemplares Bos Taurus y Bos indicus $(12,13)$ las causas de variación de la respuesta superovulatória para animales Bos indicus, subespecie a la cual pertenece la raza Brahman, corresponden a las mismas descritas para hembras donantes de origen Bos taurus $(14,15)$. Por otro lado, reportes que describan los efectos de la implementación de la ablación folicular en protocolos de SPO y que hayan sido probados en animales de la raza Brahman, son escasos en la literatura. Teniendo en cuenta lo anterior, y basados en la necesidad de generar alternativas que contribuyan a mejorar la eficiencia de la técnica de SPO y la producción in vivo de embriones, y principalmente que favorezcan la estabilización de los resultados, el presente estudio tuvo como objetivo determinar el efecto de la ablación folicular en el inicio de un protocolo de SPO sobre la respuesta superovulatoria de vacas donantes de raza Brahman.

\section{MATERIALES Y MÉTODOS}

Sitio del estudio. El presente estudio se realizó en la hacienda Oklahoma, localizada en el municipio de San Alberto, Cesar (Latitud: 7.7627, Longitud: $-73.39317^{\circ}$ $45^{\prime} 46^{\prime \prime}$ Norte, $73^{\circ} 23^{\prime} 35^{\prime \prime}$ Oeste), con altitud de $125 \mathrm{~m}$. s. n. m., temperatura promedio de $28.1^{\circ} \mathrm{C}$ y un régimen anual de lluvias de $2223 \mathrm{~mm}$ (16).

Animales. Se utilizaron 20 vacas no lactantes multíparas de la raza Brahman, con peso promedio de $510 \pm 50 \mathrm{~kg}$, edad promedio de $7 \pm 2$ años, condición corporal promedio de $3.5 \pm 1$ [escala de 1 a 5 según lo descrito por Pereira et al (17)], y ausencia de alteraciones visibles en el tracto reproductivo. Los animales fueron expuestos a las mismas condiciones ambientales, nutricionales (agua, sal mineralizada, y pastoreo ad libitum), de manejo, selección y administración de los tratamientos. Las 20 vacas fueron distribuidas aleatoriamente en dos grupos (grupo control, $\mathrm{n}=10$ y grupo ablación, $\mathrm{n}=10$ ).

Grupos experimentales y protocolos de superovulación. Previo al inicio del protocolo de SPO, a fin de confirmar la ciclicidad ovárica (presencia de un cuerpo lúteo), fue realizada una evaluación ginecológica mediante ultrasonografía vía transrectal, utilizando transductor multifrecuencia de 5 a $8 \mathrm{MHz}$ (Mindray DP 2200 vet; Mindray, Shenzhen, China).

Grupo control (G1). Se consideró como día cero (D0) el momento de la inserción de un dispositivo intravaginal impregnado con $1.0 \mathrm{~g}$ de progesterona (P4) en asociación con una inyección de benzoato de estradiol (BE) equivalente a $2.5 \mathrm{mg}$, intramuscular (im); al cuarto día de instaurado el protocolo, se inició el tratamiento de SPO mediante aplicaciones de la hormona folículo estimulante porcina (FSHp). Para este propósito, una dosis total de $200 \mathrm{mg}$ de FSHp fue dividida en ocho dosis decrecientes, distribuidas en dos aplicaciones al día en esquema am/ pm durante cuatro días $(40,40,30,30,20,20,10$ y $10 \mathrm{mg}$, im respectivamente). Concomitante con la quinta y sexta aplicación de FSHp (día 6 del protocolo), los animales recibieron dos dosis de prostaglandina F2a (PGF2a), cada una correspondiente a $25 \mathrm{mg}$, im de Dinoprost trometamina, adicionalmente, el dispositivo intravaginal fue retirado junto con la sexta aplicación de FSHp, y finalmente, 36 horas posterior a la retirada del dispositivo de P4 (día 8 del protocolo), fueron administrados 0.021 $\mathrm{mg}$, im de acetato de buserelina $(\mathrm{GnRH})$. El diseño esquemático de los grupos experimentales se puede observar en la figura 1.

Grupo ablación (G2). Los animales de éste grupo fueron inicialmente sometidos a una ablación folicular, momento que se consideró como el D0 del protocolo. La ablación consistió en la aspiración mecánica de los folículos ováricos $\geq 5 \mathrm{~mm}$, utilizando un equipo de ultrasonido (Mindray DP 2200 vet; Mindray, Shenzhen, China), 
acoplado a un transductor micro-convexo multifrecuencia de 5 a $8 \mathrm{MHz}$, el cual se ajustó a una sonda guía para ultrasonografía transvaginal utilizada en procedimientos de aspiración folicular (WTA, Cravinhos, Brasil). A través de la guía se colocó el sistema de aspiración que consistió en una aguja descartable de $20 \mathrm{G} \times 2^{3 / 4^{\prime \prime}}$ la cual se comunicó, por medio de una línea de aspiración, a un tubo plástico de $50 \mathrm{~mL}$ (Falcon, Becton Dickinso Labware, New York, USA). La presión de vacío fue generada con ayuda de una jeringa descartable de $50 \mathrm{~mL}$. El procedimiento de ablación folicular fue realizado de acuerdo con lo descrito por Acosta et al (18), de la siguiente manera: inicialmente, el área superior del espacio de las primeras vertebras coccígeas se desinfectó con solución de alcohol al $70 \%$, y en esta región se inyectaron $5 \mathrm{~mL}$ de clorhidrato de lidocaína al $2 \%$.

La vulva y región perineal se lavaron y desinfectaron con solución yodada y alcohol al $70 \%$. Posteriormente, la guía de aspiración folicular fue introducida hasta el fondo del saco vaginal y después de fijar el ovario contra el transductor por manipulación a través del recto, la aguja se desplazó hasta perforar la pared vaginal y entrar al estroma ovárico. 24 horas después de realizada la ablación folicular (D1 del protocolo) se inició el tratamiento de SPO, siguiendo las dosis, productos y esquema de aplicaciones descrito para el G1. Brevemente, para el G2 el implante intravaginal de progesterona fue colocado el DO, inmediatamente después de realizada la ablación, el tratamiento de SPO con FSHp fue administrado durante los días uno al cuatro del protocolo, el día tres (D3) fueron aplicadas las dos dosis de PGF2a (esquema am/pm) y junto a la segunda dosis de PGF2a (D3, pm) fue retirado el implante, finalmente, la aplicación del análogo de GnRH fue realizada el día cinco (D5), (Figura 1).
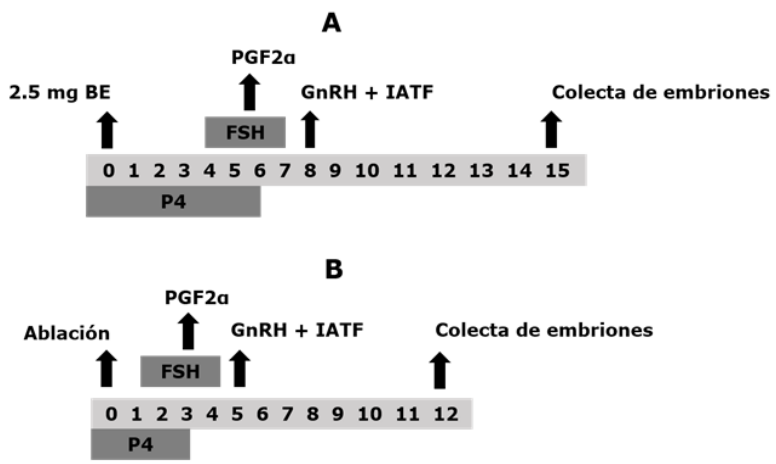

Figura 1. Diseño esquemático de los grupos experimentales. Grupo control ( $\mathrm{G} 1 ; \mathrm{n}=10, \mathrm{~A})$ y Grupo ablación (G2; $n=10, B)$. Benzoato de estradiol (BE), Prostaglandina f2a (PGF2 $\alpha$ ), Hormona liberadora de gonadotropinas (GnRH), Inseminación artificial a tiempo fijo (IATF), Hormona folículo estimulante (FSH) y progesterona (P4).

Para los dos grupos, en total fueron llevadas a cabo tres inseminaciones artificiales (IA) en cada animal, la primera siendo realizada al momento de la inyección de GnRH (D8 para el G1 y D5 para el G2), y las dos dosis restantes efectuadas con intervalos de 12 horas a partir de la primera IA. En cada IA fue utilizada una dosis de semen de 40 millones de espermatozoides en pajillas de $0.5 \mathrm{CC}$, la cual fue descongelada a $36^{\circ} \mathrm{C}$ durante 40 segundos, y con el uso de la pistola de inseminación, el contenido seminal fue depositado en el cuerpo del útero. Para todos los casos fueron utilizadas pajillas correspondientes a un mismo animal de la raza Brahman y a un mismo lote de congelación. Finalmente, La colecta de los embriones fue realizada siete días después de la primera IA (día 15 del protocolo para el grupo G1 y día 12 para el grupo G2, Figura 1).

Colecta de embriones. Previo al procedimiento, la zona perineal de las vacas donantes fue lavada y desinfectada, y se indujo anestesia epidural mediante la inyección de 5 $\mathrm{mL}$ de lidocaína al $2 \%$. La recuperación de los embriones se realizó a través de lavado uterino por gravedad con circuito cerrado, usando medio tampón salino fosfatado (PBS) suplementado con albumina sérica bovina (BSA) al $2 \%$ y antibióticos (Bioniche animal health inc; Canada). La búsqueda y clasificación de los embriones fue realizada a través de un estéreo-microscopio (Nikon SMZ645, Nikon, Japón) equipado con platina térmica a $37^{\circ} \mathrm{C}$. Para el mantenimiento de las estructuras durante el periodo de evaluación fue utilizado medio holding compuesto de PBS suplementado con BSA al $20 \%$ y antibióticos (Bioniche animal health).

La calidad de los embriones obtenidos se evaluó de forma subjetiva de acuerdo con las características morfológicas de las estructuras, siguiendo los parámetros descritos por la Sociedad Internacional de Tecnologías Embrionarias (IETS) (19), de la siguiente manera: Embriones calidad 1: (excelentes o buenos) desarrollo correspondiente al día de la colecta, blastómeros de color, tamaño, y textura uniforme, de forma esférica y zona pelúcida intacta. Al menos el $85 \%$ del material de la masa celular está intacto. Embriones calidad 2: (regular) presencia de irregularidades en la masa celular, el color o el tamaño de las células, presencia de vesiculaciones y blastómeros sueltos. Por lo menos el $50 \%$ de la masa celular está intacta. Embriones calidad 3: (malo) presencia de defectos severos en la masa celular, blastómeros sueltos, células degeneradas de diferente tamaño y numerosas vesículas. El $25 \%$ de la masa celular permanece intacta. Embriones calidad 4: (muertos o degenerados) blastómeros desorganizados, degenerados y sueltos, con células de apariencia vesicular, granular y de crecimiento retardado en relación con el día de desarrollo.

Análisis estadístico. El análisis estadístico se realizó con ayuda del programa estadístico SAS 9.4 (Institute Inc. Cary, NC, USA) Utilizando la prueba T de Student para el total de estructuras colectadas y el total de embriones transferibles por animal. La prueba Shapiro-wilk se utilizó para evaluar la distribución normal de los datos, cuando los parámetros no mostraron una distribución normal, se realizó una transformación logarítmica para una mejor homogeneidad y distribución de los residuos. la prueba de Chi cuadrado se usó para analizar la proporción de embriones degenerados, embriones de calidad 1 , calidad 2 y estructuras infértiles con relación al total de estructuras colectadas. Los valores de P inferiores a 0.05 se consideraron significativos.

Aspectos éticos. Teniendo en cuenta las directrices y normas éticas para la realización de procedimientos de investigación con animales, todos los procedimientos experimentales realizados en el presente estudio fueron aprobados por el Comité de ética para la investigación de la Universidad Pedagógica y Tecnológica de Colombia (UPTC) mediante el Acta No. 05 del 20 de marzo de 2018. 


\section{RESULTADOS}

No fueron observadas diferencias significativas entre los grupos evaluados con respecto al total de estructuras colectadas por animal $(p=0.502)$ y total de embriones transferibles por animal ( $p=0.201$, Tabla 1$)$.

Sin embargo, vacas donantes sometidas a ablación folicular produjeron una mayor proporción de embriones de calidad 1 en comparación a los animales del grupo control $(p<0.01)$. En cuanto que un mayor porcentaje de embriones calidad $2(p=0.01)$ fue obtenido de animales del grupo control (Tabla 1). Asimismo, se observó un mayor porcentaje de embriones degenerados y oocitos no fertilizados en el grupo control ( $p>0.05$, Tabla 1$)$.

Tabla 1. Respuesta superovulatoria de vacas donantes de raza Brahman a protocolos hormonales de superovulación (SPO). Grupo control y grupo ablación (ablación folicular previo al tratamiento de SPO).

\begin{tabular}{|c|c|c|c|}
\hline \multirow{2}{*}{ Variable } & \multicolumn{2}{|c|}{ Grupos experimentales } & \multirow{2}{*}{ Valor-P } \\
\hline & Control & Ablación & \\
\hline $\begin{array}{l}\text { Total estructuras colectadas/ } \\
\text { animal }{ }^{+}\end{array}$ & $\begin{array}{l}11.85 \\
\pm 1.96\end{array}$ & $\begin{array}{l}12.10 \\
\pm 1.47\end{array}$ & 0.502 \\
\hline Oocitos no fertilizados, $\%(n / n)^{*}$ & $\begin{array}{c}6.93 \\
(16 / 235)\end{array}$ & $\begin{array}{c}2.88 \\
(7 / 243)\end{array}$ & 0.040 \\
\hline Embriones degenerados, $\%(n / n)^{*}$ & $\begin{array}{c}18.14 \\
(43 / 235)\end{array}$ & $\begin{array}{c}10.33 \\
(25 / 243)\end{array}$ & 0.014 \\
\hline Embriones calidad $1, \%(n / n)^{*}$ & $\begin{array}{c}31.22 \\
(74 / 235)\end{array}$ & $\begin{array}{c}68.60 \\
(166 / 243)\end{array}$ & $<0.01$ \\
\hline Embriones calidad $2, \%(n / n)^{*}$ & $\begin{array}{c}43.04 \\
(102 / 235)\end{array}$ & $\begin{array}{c}18.60 \\
(45 / 243)\end{array}$ & $<0.01$ \\
\hline Embriones transferibles/animal ${ }^{+}$ & $\begin{array}{l}8.8 \\
\pm 1.24\end{array}$ & $\begin{array}{c}10.5 \\
\pm 1.14\end{array}$ & 0.201 \\
\hline
\end{tabular}

*Valores ( $n / n)$ corresponden al número de cada variable, con relación al total de estructuras. +Valores representan promedio \pm desviación estándar. Los valores de $p<0.05$ se consideraron significativos.

\section{DISCUSIón}

En este estudio fueron evaluados dos métodos de sincronización de la emergencia de una nueva onda folicular para propósitos de SOP y TE, pudiendo ser designados, de modo general, como un método físico y otro farmacológico. Sin embargo, independiente del método, la tasa de recuperación de embriones

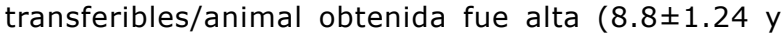
$10.5 \pm 1.14)$. Según la IETS $(20)$ en Suramérica se producen en promedio 7.5 embriones transferibles/ colecta y en el mundo se producen 6.7 embriones transferibles/colecta, datos inferiores a los observados en el presente estudio.

Determinados factores que fueron considerados como criterios de selección para las donantes que participaron del experimento, tales como la ciclicidad ovárica (presencia de cuerpo lúteo), adecuada condición corporal, edad y estado productivo (no lactante), e incluso la subespecie a la cual pertenecen, podrían haber influenciado este resultado. Particularmente, hembras bovinas de origen Bos indicus presentan elevados concentraciones circulantes de factor de crecimiento similar a insulina (IGF) en comparación a animales Bos Taurus, esta característica ha sido relacionada con una población folicular elevada propia de hembras de esta subespecie (21), por consiguiente, al trabajar con vacas de la raza Brahman, una considerable cantidad de folículos se encuentran disponibles y con posibilidad de responder adecuadamente a los tratamientos de SPO $(13,22)$

Al comparar las dos metodologías, fue observado que el uso de la ablación folicular aumentó el porcentaje de embriones de calidad 1 . Ha sido ampliamente demostrado que la ausencia de un folículo dominante al inicio de la estimulación del crecimiento folicular con FSH exógena, es uno los factores que mayor influencia tienen en la respuesta superovulatoria, no solamente en lo referente al número de ovulaciones, sino además en la calidad de las estructuras recuperadas $(4,23)$.

El uso de la combinación de los esteroides P4 y E2 para sincronizar el inicio de una onda de crecimiento folicular se basa en el efecto supresor de la P4 sobre la liberación de gonadotropinas hipofisiarias principalmente sobre la $\mathrm{LH}$, unido a la atresia folicular causada por el E2, a través del efecto transitorio de supresión de las concentraciones de FSH (7). Una vez que las concentraciones de E2 comienzan a disminuir, como consecuencia de la metabolización de esta molécula por el organismo del animal, las concentraciones de FSH aumentan, permitiendo de esta forma la aparición de una nueva onda de crecimiento folicular (3). Sin embargo, ha sido observado que en ocasiones estos tratamientos fallan en la supresión de los folículos dominantes, permitiendo que estos persistan aún después del tratamiento y que generen un efecto inhibitorio sobre el desarrollo de los demás folículos (24). Asimismo, folículos dominantes funcionales presentes antes y al inicio del tratamiento con P4 y E2 pueden haber ejercido diferentes grados de atresia sobre el resto de la población folicular (24). En cualquiera de los casos, dependiendo del grado de atresia sufrido por cada folículo, la calidad de los oocitos contenidos se afecta de forma variable, repercutiendo en la calidad final de los embriones generados después del proceso de fecundación (25). Por su parte, la remoción mecánica de los folículos de mayor tamaño $(\geq 5 \mathrm{~mm}$ ) podría ser un método más efectivo para impedir la presencia de folículos funcionalmente dominantes, resultando en un grupo más homogéneo de pequeños folículos renovados que crecen de forma sincrónica por efecto de la FSH exógena, en consecuencia, oocitos con mayor grado de competencia serían ovulados al final del tratamiento $(23,27)$.

Los mecanismos asociados al efecto que las células foliculares tienen sobre la competencia de los oocitos, se relacionan con la actividad que ejercen diversas sustancias de distinta naturaleza (factores de crecimiento, microRNAs, aminoácidos, ácidos grasos, carbohidratos, entre otros) y que son producidas de forma paracrina o liberadas a través de vesículas secretoras en sentido bidireccional, con el objetivo de coordinar el adecuado desarrollo de los oocitos en crecimiento (26). En ese sentido, la viabilidad de las células foliculares es requisito esencial para asegurar la apropiada comunicación entre estos tipos celulares, y así garantizar la adquisición de competencia de desarrollo posterior del oocito.

Con relación a las variables, oocitos infértiles y embriones degenerados, se observó una mayor proporción de estas estructuras para el grupo control. Estos resultados podrían estar asociados a los mismos factores discutidos anteriormente, que habrían influenciado en la calidad 
de los oocitos y, por ende, en la proporción de oocitos fecundados y en la calidad de los embriones obtenidos (25). Sin embargo, para estas variables, Baracaldo et al (10) y Surjus et al (27), no encontraron diferencias entre la implementación o no de la ablación folicular. La cantidad de oocitos no fecundados e inclusive el potencial de desarrollo normal de los embriones también dependen de la capacidad fértil de los espermatozoides utilizados para el proceso de IA (28). La presencia de alteraciones en las células espermáticas, principalmente las asociadas con presencia de puntos de fragmentación del ADN, han sido relacionadas con fallas en las subsecuentes divisiones celulares de los blastómeros, que de acuerdo al grado de fragmentación, podrían resultar en desarrollo embrionario inadecuado, o inclusive, fallas desde el propio proceso de fecundación (29). Diferencias en la calidad del material seminal utilizado para las IA en los diferentes trabajos, podrían explicar la divergencia en los resultados.

En el presente estudio no se detectaron diferencias entre los dos grupos para las variables, total de estructuras colectadas y total de embriones transferibles por animal. De forma similar, Baracaldo et al (10) y Surjus et al (27) reportaron que aun cuando el número de embriones colectados aumentó en respuesta a la ablación, no hubo diferencia en la cantidad de embriones transferibles cuando esta técnica fue comparada al protocolo farmacológico convencional. La cantidad de estructuras colectadas, y dentro de estas, el total de embriones producidos, corresponden a variables influenciadas de forma directa por el tamaño de la población de folículos presentes en los animales tratados y por la respuesta ovulatoria de estas estructuras a los tratamientos hormonales, siendo características independientes de la calidad oocitaria, cuando ésta no se encuentra altamente comprometida (30).
En conclusión, el uso de ablación folicular aumentó el porcentaje de embriones de calidad tipo 1, sugiriendo que la implementación de dicha técnica podría sustituir el tratamiento tradicional con esteroides al inicio de los tratamientos de SPO, pudiendo además resultar en mejores porcentajes de preñez y superiores tasas de sobrevivencia embrionaria post criopreservación en programas comerciales de TE. Más estudios son necesarios para determinar los efectos de la ablación folicular en la respuesta superovulatoria. La inclusión de análisis moleculares donde se evalúen la calidad oocitaria y embrionaria podrían ayudar en el entendimiento de los mecanismos que son alterados por el proceso de ablación previo al inicio de protocolos de SPO.

\section{Conflicto de intereses.}

La hacienda Oklahoma suministró los animales para la realización del estudio. La hacienda Oklahoma no desempeño ningún papel en el diseño del estudio ni en la recopilación análisis e interpretación de los datos, ni en la decisión de presentar el manuscrito para su publicación. Ninguno de los autores tiene relaciones financieras o personales que puedan influir o sesgar de manera inapropiada el contenido del artículo.

\section{Agradecimientos}

Ganadería Oklahoma, empresa donde se llevaron a cabo los procedimientos con los animales. A la Dra. Carolina Jacometo Bespalhok por la traducción del documento.

\section{REFERENCIAS}

1. Baruselli PS, Ferreira RM, Sales JNS, Gimenes LU, Sá Filho MF, Martins CM, et al. Timed embryo transfer programs for management of donor and recipient cattle. Theriogenology. 2011; 76(9):1583-1593. DOI: https://doi.org/10.1016/j. theriogenology.2011.06.006

2. Blondin P. Status of embryo production in the world. Anim Reprod. 2015; 12(3) 356-358. DOI: https:// doi.org/10.1080/09613218.2015.993536

3. Bó GA, Carballo D, Tríbulo A, Tríbulo H, Tríbulo R, Rogan D, et al. New approaches to superovulation in the cow. Reprod Fertil Dev. 2009; 22(1):106-112. DOI: https://doi.org/10.1071/RD09226

4. Kohram $\mathrm{H}$ and Poorhamdollah M. Relationships between the ovarian status and superovulatory responses in dairy cattle. Anim Reprod Sci. 2012; 131(3-4):123-128. DOI: https://doi.org/10.1016/j. anireprosci.2012.03.004

5. Hasler JF. Forty years of embryo transfer in cattle: A review focusing on the journal Theriogenology, the growth of the industry in North America, and personal reminisces. Theriogenology. 2014; 88(1): 152-169. https://doi.org/10.1016/j. theriogenology.2013.09.010
6. Mapletoft RJ, Bó GA. The evolution of improved and simplified superovulation protocols in cattle. Reprod Fertil Dev. 2011; 24(1):278-283. DOI: https://doi. org/10.1071/RD11919

7. Mapletoft RJ, Bó GA. Innovative strategies for superovulation in cattle. Anim Reprod. 2013; 10(3):174-179. http://www.cbra.org.br/portal/ downloads/publicacoes/animalreproduction/issues/ download/v10n3/p174-179\%20(AR613).pdf

8. Bó GA, Mapletoft RJ. Historical perspectives and recent research on superovulation in cattle. Theriogenology. 2014; 81(1):38-48. DOI: https:// doi.org/10.1016/j.theriogenology.2013.09.020

9. Aslan S, Arslanbas D, Beindorff N, Bollwein $H$. Effects of Induction of Ovulation with $\mathrm{GnRH}$ or hCG on Follicular and Luteal Blood Flow in Holstein-Friesian Heifers. Reprod Dom Anim. 2011; 46(5):781-786. DOI: https://doi.org/10.1111/ j.1439-0531.2010.01741.x

10. Baracaldo MI, Martinez M, Adams GP, Mapletoft RJ. Superovulatory response following transvaginal follicle ablation in cattle. Theriogenology. 2000; 53(6):1239-1250. DOI: https://doi.org/10.1016/ $\underline{\mathrm{S} 0093-691 \times(00) 00268-5}$ 
11. Honparkhe M, Gandotra VK, Matharoo JS, Ghuman SPS, Dadarwal D, Jaswant Singh. Synchronization of follicular wave emergence following ultrasoundguided transvaginal follicle ablation or estradiol-17 $\beta$ administration in water buffalo (Bubalus bubalis). Anim Reprod Sci. 2014; 146(1-2):5-14. DOI: https://doi.org/10.1016/j.anireprosci.2014.02.006

12. Sartori R, Bastos M, Baruselli PS, Gimenes L, Ereno R, Barros CM. Physiological differences and implications to reproductive management of Bos taurus and Bos indicus cattle in a tropical environment. Soc Reprod Fertil Sup. 2010; (67):357-375. DOI: https://www.ncbi.nlm.nih.gov/ pubmed/21755684

13. Silva-Santos KC, Siloto LS, Santos GMG, Morotti F, Marcantonio TN, Seneda MM. Comparison of Antral and Preantral Ovarian Follicle Populations between Bos indicus and Bos indicus taurus Cows with High or Low Antral Follicles Counts. Reprod Dom Anim. 2013; 49(1):48-51. DOI: https://doi.org/10.1111/ rda. 12222

14. Soares JG, Martins CM, Carvalho NAT, Nicacio AC, Abreu-Silva AL, Campos EP, et al. Timing of insemination using sex-sorted sperm in embryo production with Bos indicus and Bos Taurus superovulated donors. Anim Reprod Sci. 2011; 127(3-4):148-153. DOI: https://doi.org/10.1016/j. anireprosci.2011.08.003

15 Sartori R and Barros CM. Reproductive cycles in Bos indicus cattle. Anim Reprod Sci. 2011; 124(34):244-250. DOI: https://doi.org/10.1016/j. anireprosci.2011.02.006

16. Climate-data. Historical average temperatura. Clima San Alberto [en línea]. Climate-data; 2018; https:// es.climate-data.org/america-del-sur/colombia/ cesar/san- alberto-34400/

17. Pereira LL, Ferrreira AP, Vale WG, Sequire LR, Neves $K A L$, Morini AC, et al. Effect of body condition score and reuse of progesterone-releasing intravaginal devices on conception rate following timed artificial insemination in nelore cows. Reprod Dom Anim. 2018; 53(3):624-628. DOI: https://doi. org/10.1111/rda.13150

18. Acosta DAV, Rivelli MI, Skenandore C, Zhou Z, Keisler DH, Luchini D, et al. Effects of rumenprotected methionine and choline supplementation on steroidogenic potential of the first postpartum dominant follicle and expression of immune mediators in Holstein cows. Theriogenology. 2017; 96:1-9. DOI: https://doi.org/10.1016/j. theriogenology.2017.03.022

19. Robertson I and Nelson RE. Certification and identification of embryos; In: Stringfellow $D$ and Givens MD, editor. Fourth edition of manual of the international embryo transfer society (IETS). Champaign, Illinois 61822 USA. 2010; 95-108.

20. Perry G. 2016 statistics of embryo collection and transfer in domestic farm animals. International embryo technology society (IETS) data retrieval committee. [En linea]. 2016. https://www. iets.org/pdf/comm data/IETS Data Retrieval Report_2016_v2.pdf
21. Satrapa RA, Razza EM, Castilho ACS, Simões RAL, Silva CF, Nabhan T, et al. Differential Expression of IGF Family Members in Heat-Stressed Embryos Produced In Vitro from OPU-Derived Oocytes of Nelore (Bos indicus) and Holstein (Bos taurus) Cows. Reprod Dom Anim. 2013; 48(6):1043-1048. DOI: https://doi.org/10.1111/rda.12211

22. Batista EOS, Macedo GG, Sala RV, Ortolan MDDV, Sá Filho MF, Del Valle TA, el at. Plasma Antimullerian Hormone as a Predictor of Ovarian Antral Follicular Population in Bos indicus (Nelore) and Bos Taurus (Holstein) Heifers. Reprod Dom Anim. 2014; 49(3):448-452. DOI: https://doi.org/10.1111/ rda. 12304

23. Amiridis GS, Tsiligianni T, Vainas E. Follicle Ablation Improves the Ovarian Response and the Number of Collected Embryos in Superovulated Cows During the Early Stages of Lactation. Reprod Dom Anim. 2006; 41(5):402-407. DOI: https://doi. org/10.1111/j.1439-0531.2006.00684.x

24. Ginther JO and Hoffman MM. Intraovarian effect of dominant follicle and corpus luteum on number of follicles during a follicular wave in heifers. Theriogenology. 2014; 82(1):169-175. DOI: https:// doi.org/10.1016/j.theriogenology.2014.03.013

25. Baruselli PS, Sa Filho MF, Ferreira RM, Sales JNS, Gimenes LU, Vieira LM, Mendanha MF, Bo GA. Manipulation of follicle development to ensure optimal oocyte quality and conception rates in cattle. Reprod Dom Anim. 2012; 47(4): 134-141. DOI: https://doi.org/10.1111/j.1439$\underline{0531.2012 .02067 . x}$

26. Nuttinck F. Oocyte related factors impacting on embryo quality: relevance for in vitro embryo production. Anim Reprod. 2018; 15(3):271-277. DOI: https://doi.org/10.21451/1984-3143AR2018-0077

27. Surjus RS, Prata $A B$, Borsato $M$, Mattos $F$, Martins da Silveira MC, Mourão GB, et al. In vivo embryo production in cows superovulated 1 or 2 days after ovum pick-up. Reprod Fertil Dev. 2013; 26(4):527532. DOI: https://doi.org/10.1071/RD12398

28. Samardzija M, Getz I, Lojkic M, Valpotic H, Djuricic D. Optimization of Sperm for In vitro Production of Bovine Embryos. SOJ Vet Sci. 2015; 1(2):1-7. DOI: http://dx.doi.org/10.15226/2381-2907/1/2/00107

29. Simon L, Murphy MB, Shamsi L, Liu B, Emery KI, Aston J, et al. Paternal influence of sperm DNA integrity on early embryonic development. Hum Reprod. 2014; 29(11):2402-2412. DOI: https:// doi.org/10.1093/humrep/deu228

30. Silva SK, Santos GM, Koetz JC, Morotti F, Siloto LS Marcantonio TN, et al. Antral Follicle Populations and Embryo Production - In Vitro and In Vivo - of Bos indicus-taurus Donors from Weaning to Yearling Ages. Reprod Dom Anim. 2014; 49(2):228-232. DOI: https://doi.org/10.1111/rda.12255 